\title{
Single-donor islet transplantation in type I diabetes: patient selection and special considerations
}

\author{
Jacob A Tatum* \\ Max O Meneveau* \\ Kenneth L Brayman \\ Department of Surgery, Division \\ of Transplantation, The University \\ of Virginia Health System, \\ Charlottesville, VA, USA
}

*These authors contributed equally to this work.
This article was published in the following Dove Press journal:

Diabetes, Metabolic Syndrome and Obesity:Targets and Therapy 28 February 2017

Number of times this article has been viewed

\begin{abstract}
Type 1 diabetes mellitus is an autoimmune disorder of the endocrine pancreas that currently affects millions of people in the United States. Although the disease can be managed with exogenous insulin administration, the ultimate cure for the condition lies in restoring a patient's ability to produce their own insulin. Islet cell allotransplantation provides a means of endogenous insulin production. Though far from perfected, islet transplants are now a proven treatment for type 1 diabetics. However, proper patient selection is critical for achieving optimal outcomes. Given the shortage of transplantable organs, selecting appropriate candidates for whom the procedure will be of greatest benefit is essential. Although many of those who receive islets do not retain insulin independence, grafts do play a significant role in preventing hypoglycemic episodes that can be quite detrimental to quality of life and potentially fatal. Additionally, islet transplant requires lifelong immunosuppression. Antibodies, both preformed and following islet infusion, may play important roles in graft outcomes. Finally, no procedure is without inherent risk and islet transfusions can have serious consequences for recipients' livers in the form of both vascular and metabolic complications. Therefore, patient-specific factors that should be taken into account before islet transplantation include aims of therapy, sensitization, and potential increased risk for hepatic and portal-venous sequelae.
\end{abstract}

Keywords: islet transplantation, diabetes mellitus type 1, brittle diabetes, single donor, patient

\section{Introduction}

Type 1 diabetes mellitus (DM1) affects nearly three million Americans. ${ }^{1}$ It is a challenging disease to manage, often requiring complex medical regimens and significant effort from both the clinician and the patient. DM1 is characterized by autoimmune destruction of insulin-producing beta cells. The first manifestation of beta cell loss is loss of first-phase insulin secretion (peak levels of insulin in the minutes following a glucose challenge) and is then followed by widespread destruction of beta cells and persistent occurrence of hyperglycemia. ${ }^{2}$ This destruction is mediated by a host of immune factors, including beta-cell autoantigens, dendritic cells, macrophages, B lymphocytes, and T lymphocytes. ${ }^{3}$ The resulting insulin deficiency causes a state of glucose dysregulation, requiring exogenous insulin administration and careful glucose monitoring. Despite optimal therapy, however, a subset of patients still experience unmanageable and unpredictable fluctuations in their blood glucose. ${ }^{4}$

The two most promising techniques for restoring endogenous insulin production have been whole pancreas transplantation and islet-cell transplantation. Islet transplant is significantly less invasive than pancreatic transplant and has been shown to significantly reduce the burden of exogenous insulin requirement. Islet transplantation has
Correspondence: Kenneth L Brayman Department of Surgery, University of Virginia Health System, 1215 Lee Street, Charlottesville, VA 22908, USA

Tel +l 4349249370

Fax + I 4349245539

Email klb9r@virginia.edu 
also been shown to slow or even halt the progression of serious diabetic sequelae such as cardiovascular complications, retinopathy, nephropathy, and neuropathy. ${ }^{5,6}$

In islet transplantation, pancreatic tissue from a donor is processed and islet cells are isolated. A single healthy human pancreas can contain 4-15 million islets. ${ }^{7}$ Isolation and purification is done using a combination of enzymatic tissue digestion as well as mechanical agitation. The yield and purity of islets remains highly variable with some studies showing an islet equivalents count ranging from 100,000 to 300,000 per pancreas (islet equivalent: a standardized value of islet volume). ${ }^{8}$ The isolated cells are then injected into the hepatic portal system of the recipient with the aim of producing a viable donor islet-cell population in the liver.

To establish an islet population capable of producing enough insulin for clinical effect, a large number of these cells $\left(>5,600\right.$ islet equivalents $/ \mathrm{kg}$ ) must be transplanted..$^{9}$ Post-transplant, the C-peptide level is used as a marker for graft survival as its production often outlasts insulin independence and overall clinical effect of the transplant. Oftentimes patients require multiple infusions from multiple donors over time to achieve sufficient endogenous insulin production. A drawback of this method is the chance of recipient sensitization, as they are exposed to such a large number of donor antigens. ${ }^{9}$ Thus, the ideal method is one infusion of islets from one single donor. Additionally, limiting the number of required islet transfusions helps mitigate the cost, improves access to transplantation, and limits the burden of this procedure over time.

Not all patients with DM1 are good candidates for islet cell transplantation. For example, characteristics such as glucose lability, preexisting insulin resistance, obesity, sensitization, and the presence of beta-cell autoantibodies affect the success and appropriateness of this intervention. Given the limits of islet availability and the significant burden that comes with receiving a transplant, selecting appropriate candidates for islet transplantation is essential. Here, a number of important aspects and considerations in patient selection for islet transplantation are reviewed.

\section{Brief history of islet transplantation}

The knowledge that pancreatic dysfunction causes a syndrome similar to DM1 dates back to 1889 when von Mering and Minkowski discovered that pancreatectomy lead to hyperglycemia, ketoacidosis, and death. ${ }^{10}$ Despite significant improvements in the understanding of DM1 throughout the 20th century, it was not until 1977 that the development of a clinically successful cellular replacement therapy emerged.
Using the newly discovered azathioprine and corticosteroids as immunosuppression, Najarian et al described the first successful islet cell transplant. ${ }^{11}$ Three years later, Largiadèr et al demonstrated the first case of insulin independence after islet allotransplantation. ${ }^{12}$ Overall, however, outcomes from islet transplantation remained significantly worse than those of solid organ transplantation until the description of the socalled Edmonton protocol in $2000 .^{13}$

This landmark protocol provided a standardized procedure by which islet transplantation could be safely and effectively performed and could dramatically improve outcomes. ${ }^{13}$ In particular, the two most notable changes introduced by the Edmonton protocol were the use of multiple islet transplantations to increase the mass of cells provided, as well as shifting immunosuppression away from steroids and using low-dose anti-interleukin 2 and sirolimus to minimize betacell toxicity. Since the Edmonton protocol, significant work has been done to improve islet transplantation. ${ }^{14}$ Despite this, islet transplant remains relatively rare, with only 44 islet transplant centers currently active in the world. ${ }^{15}$ As islet transplantation becomes more common and more centers begin adopting it as a therapy for type 1 diabetics, the need for defining optimal recipient characteristics becomes ever more important.

\section{Patient selection}

Like any surgical procedure, islet transplantation carries inherent risks. Additionally, the postoperative immunosuppressive regimen can cause significant morbidity and lifestyle challenges. Therefore, appropriate selection of candidates is paramount. The following paragraphs address common considerations for evaluating which patients would benefit most from single donor islet transplantation. Common indications and exclusion criteria for islet transplantation are summarized in Box 1.

\section{Labile diabetes}

The goal of islet and pancreas transplantation is to eliminate the requirement for exogenous insulin in type 1 diabetic patients. However, 5-year insulin independence has been difficult to achieve. Currently $82 \%$ of patients receiving pancreas transplant are insulin independent at 1 year and $58 \%$ at 5 years. ${ }^{16}$ Islet transplantation has been less successful at providing durable insulin independence, with only $66 \%$ of patients remaining off insulin at 1 year, and $44 \%$ at 3 years. ${ }^{17}$ Interestingly, in a number of recent studies, patients who received islet transplants but failed to remain insulin independent had significantly reduced incidence 


\section{Common indications for islet transplantation}

Type 1 diabetes for $>5$ years

Negative stimulated C-peptide level

Hypoglycemic unawareness

Severe hypoglycemic episodes despite optimal therapy

Glycemic lability

\section{Common exclusions for islet transplantation}

Severe cardiovascular disease

Insulin resistance

GFR $<80$

Portal hypertension

Active or chronic hepatitis B or C

Inability to comply with immunosuppression

Box I Common indications and exclusions for islet transplantation

Abbreviation: GFR, glomerular filtration rate.

of hypoglycemic events post-transplant. ${ }^{18}$ This was most recently demonstrated in a phase 3 trial by the Clinical Islet Transplantation Consortium. ${ }^{19}$ This trial demonstrated that patients had a $93 \%$ probability of being free from hypoglycemic events at 2 years post-transplant. Though islet transplant is less successful than pancreas transplantation at maintaining insulin independence, these findings suggest a role for islet transplant in patients with labile diabetes. In other words, this therapy could best serve those patients whose greatest impediment to a normal life is not insulin administration, but unpredictable and often debilitating hypoglycemic episodes and hypoglycemic unawareness.

The volume of infused islets correlates to the duration of insulin independence post-transplant. ${ }^{20}$ Therefore, a barrier to islet transplantation has been the need for recurrent islet infusions due to decreasing graft function over time. ${ }^{21,22}$ However, a French study of 23 patients showed preserved hypoglycemic protection despite decreasing allograft function as measured by the $\beta$-score (a validated score for describing overall graft function). ${ }^{23,24}$ In other words, transplanted patients were protected against hypoglycemic events despite decreasing allograft function over time. Subsequent trials have been consistent with this finding. In a study of 12 islet recipients, for example, Rickels et al reported dramatic and durable reduction in glycemic variability despite the fact that many of the patients did not remain insulin independent over time. ${ }^{25}$ One explanation for this finding is that in many patients with progressive graft-failure, some latent $\mathrm{C}$-peptide production persists. This production of C-peptide indicates that beta cells, though small in number, continue to survive and protect against hypoglycemia by stimulating glucagon production in nearby alpha cells via a paracrine pathway. ${ }^{26}$

It would seem, therefore, that single donor islet transplantation can be used instead of the traditional multiple infusions for patients whose primary goal is reduction of glycemic variability and hypoglycemic unawareness. This would ultimately provide significant lifestyle benefit for brittle diabetics and expand the donor pool for those patients who would benefit from this therapy. Though these results are quite promising, it must be noted that the majority of the aforementioned trials have been very small. Further study with large prospective trials will reveal whether the initial findings are in fact applicable to the general population of type 1 diabetic patients.

\section{Immunity}

Two immune mechanisms are responsible for graft loss in islet transplant patients: 1) destruction of transplanted islet cells by the same mechanism that destroyed the native insulin-producing cells - autoantibodies; 2) graft loss due to rejection from alloimmunity. Both cellular and humoral mechanisms have been implicated in graft destruction of transplanted islet cells. Cellular immunity has been difficult to study given the inherent difficulty of sampling engrafted islets in the recipient liver. As such, humoral immunity and in particular antibody assays have been the primary area of focus. Though autoantibodies lead to the initial destruction of the islets in DM1, studies have found that pre-transplant autoantibody titers are only weakly associated with graft survival and overall outcomes. ${ }^{27}$

Although the presence of these autoantibodies pretransplant are poorly predictive of graft loss, activation of these autoimmune pathways post-transplant is associated with graft loss and eventual failure. ${ }^{28}$ A study by Piemonti et al, for example, showed that a statistically significant rise in anti-glutamic acid decarboxylase or anti-islet antigen 2 autoantibodies (antibodies primarily responsible for beta cell loss in DM1) resulted in a significantly increased risk of graft failure when compared to those without a rise in those antibodies with a relative risk of graft failure of 5.3. ${ }^{27}$ Furthermore, those patients who experienced an increase in autoantibody titer had a shorter median time to graft failure when compared to those who did not experience autoantibody increases.

Although autoimmunity is a significant aspect of DM1, islet transplantation, like most other types of transplant, is also challenged by the issue of alloimmunity or the body's reaction against foreign antigens. In addition to studying autoantibodies, Piemonti et al examined how donor-specific alloantibodies (DSA) affected graft failure in islet recipients. 
The relative risk of developing graft failure in those patients with a rise in DSA, despite adequate immunosuppression, was 5.12 when compared to those without a rise in DSA. Risk factors for increased DSA included multiple islet infusions, human leukocyte antigen (HLA) class I or II mismatch, or pre-transplant DSA. Additionally, DSA was higher in regimens where anti-thymocyte globulin was used for induction and mycophenolate mofetil was used for maintenance immunosuppression. The groups in which DSA remained low were those in which induction was done using an anti-CD25 antibody, and where sirolimus or tacrolimus were used for maintenance. ${ }^{27}$

It is clear that immunity plays a significant role in islet cell transplant outcomes. DM1 is unique in that autoantibodies play a role in the initial disease pathogenesis and also seem to affect the success of islet transplantation. It is surprising that preformed autoantibodies are only weakly associated with outcomes and that significant negative effects were noted when those antibodies increased post-transplant. Preformed autoantibody titers, therefore, are difficult to use for predicting who will and will not have a successful outcome. Currently, HLA matching is not performed in islet transplantation. ${ }^{29}$ However, HLA mismatch was associated with increased DSA and graft failure. A strategy for outcome improvement could include matching donor and recipients with HLA II match and HLA I mismatch. This would theoretically prevent autoimmune destruction through HLA I and limit the development of DSA and panel reactive antibody reactivity to class II antigens. Immunity remains a significant challenge to islet transplant outcomes, and further study will be required to characterize optimal patient-donor matching characteristics and develop improved immunosuppression that limits toxicity to engrafted islets.

\section{Special considerations}

Like any other procedure, islet transplantation is not without risk of complications. One of the particular risks of this therapy comes from the portal vein infusion process. A number of studies have shown that portal vein hypertension, thrombosis, or infarction of the liver can all occur as a result of islet infusion. Rates of thrombosis range from $0 \%$ to $3.9 \%$, and the risk of bleeding is even higher, ranging from $0 \%$ to $6.5 \%{ }^{30-33}$ At least one group performing islet transplants has been preferentially cannulating only the left branch of the portal vein to facilitate future resection should serious complications of the infusion arise. ${ }^{34}$ Given that islet infusion poses a risk to hepatic vasculature, some have proposed that there is an upper limit to the number of safe islet infusions that should be done. ${ }^{34}$ This risk must be balanced with the goals of transplantation, one of which being insulin independence, which is better achieved with multiple islet infusions.

In addition to causing vascular insult, islet infusion may also lead to hepatic steatosis. First found in animal models, it has only been recently shown that this occurs in humans after transplantation. ${ }^{35}$ Various groups have started assessing for steatosis in their postoperative cohorts using a variety of imaging modalities, most notably ultrasound and MRI. ${ }^{36,37}$ In these studies, rates of fatty liver post-transplant have ranged from $17 \%$ to $24 \%$. There was no correlation between HbA1c or body mass index and steatosis post-transplant in either study. Fat deposition tends to be transient and can vary widely throughout a patient's course. Interestingly, some patients with fat deposition tend to have better graft function, as defined by superior beta scores compared to their non-steatotic counterparts. Graft function tends to diverge late $(40+$ months), those with steatosis experiencing faster rates of graft exhaustion and increased islet loss. ${ }^{36}$ This finding is not universal, however, as at least one group has found contradictory data. ${ }^{38}$

Steatosis of hepatic parenchyma is a cornerstone of nonalcoholic fatty liver disease (NAFLD). The disease is more often associated with diabetes mellitus type 2 but is seen in those with type 1 at higher incidences than the general population. This association has been correlated to increased risk of heart disease and chronic kidney disease in these type 1 diabetics. ${ }^{39}$ The ramifications of this finding may be twofold with regard to islet transplantation. 1) Although it is well understood that diabetes in and of itself causes heart disease and kidney dysfunction, ${ }^{40}$ islet grafts that cause steatosis may in turn also lead to increased rates of heart and kidney disease. 2) That steatosis caused by islet infusion is associated with shorter graft-survival times. Although steatosis in islet transplant is only just beginning to be studied, it may hold that preexisting steatosis such as that caused by NAFLD may also speed graft loss. Therefore, it could be that those with preexisting heart disease, kidney disease, or NAFLD are far less likely to benefit from islet transplant despite the fact that the progression of these diabetic complications are slowed by islet transplantation. Though a speculation at this time, this hypothesis warrants further study as the findings may directly impact proper patient selection.

\section{Conclusion}

Though great strides have been made in islet transplantation, the treatment often falls short in its goal of producing durable, life-long insulin independence. Achieving 
long-term success with single donor islet transplantation has been difficult due to the smaller number of islets transplanted. Indeed, it has been shown that overall duration of graft survival is predicted by initial graft success. ${ }^{41}$ Long-term insulin independence has largely been achieved by infusing a large number of islets from multiple donors. Despite its shortcomings in terms of transplanted islet mass and insulin independence, single-donor islet transplant still provides significant benefit for a specific patient population: brittle diabetics. Hypoglycemic events are a source of great morbidity and mortality; these episodes can largely be avoided with islet transplant even if insulin independence is never achieved, thus providing security and improved quality of life to recipients. ${ }^{4}$ Additionally, rejection remains one of the greatest barriers to long-term graft survival. Data are beginning to emerge with regard to the role of recipients' auto- and alloimmune responses to grafts. So far, these factors have been difficult to apply to patient selection. With further study, knowledge of the immune processes specific to islet transplantation will allow superior patientdonor matching and decrease the need for postoperative immunosuppression. Some current work has focused on reducing the exposure of transplanted islets to the recipient immune microenvironment by methods such as local drug release and cellular encapsulation. ${ }^{42-44}$ Stem cell strategies for type 1 diabetes have also been considered, as obtaining sufficient beta cells for transplantation remains a significant barrier to success. Results have been mixed, but stem-cell therapy remains a promising hope for cure. ${ }^{45}$

The majority of studies examining islet transplantation have been small and generally lacking in power. As this therapy continues to improve and become more common, larger studies will be able to better characterize the important factors related to patient selection. What can be concluded now, however, is that islet transplantation remains an optimal therapy for patients with brittle diabetes and difficult-to predict hypoglycemic events.

\section{Disclosure}

The authors report no conflicts of interest in this work.

\section{References}

1. KW-T1D_Factsheet.pdf. http://www2.jdrf.org/site/DocServer/KWT1D_Factsheet.pdf?docID=1024. Accessed May 30, 2016.

2. Eisenbarth GS. Type I diabetes mellitus. N Engl J Med. 1986;314(21): $1360-1368$.

3. Yoon J-W, Jun H-S. Autoimmune destruction of pancreatic beta cells. Am J Ther. 2005;12(6):580-591.

4. Bassi R, Fiorina P. Impact of islet transplantation on diabetes complications and quality of life. Curr Diab Rep. 2011;11(5):355-363.
5. Maffi P, Secchi A. Clinical results of islet transplantation. Pharmacol Res. 2015;98:86-91.

6. Vantyghem M-C, Quintin D, Caiazzo R, et al. Improvement of electrophysiological neuropathy after islet transplantation for type 1 diabetes: a 5-year prospective study. Diabetes Care. 2014;37(6):e141-e142.

7. Ionescu-Tirgoviste C, Gagniuc PA, Gubceac E, Mardare L, Popescu I, Dima S, Militaru M. A 3D map of the islet routes throughout the healthy human pancreas. Sci Rep. 2015;5.

8. Nano R, Clissi B, Melzi R, et al. Islet isolation for allotransplantation: variables associated with successful islet yield and graft function. Diabetologia. 2005;48(5):906-912.

9. Al-Adra DP, Gill RS, Imes S, et al. Single-donor islet transplantation and long-term insulin independence in select patients with type 1 diabetes mellitus. Transplantation. 2014;98(9):1007-1012.

10. von Mering J, Minkowski O. Diabetes mellitus after pancreas extirpation. Arch Exp Pathol Pharmakol. 1889;26:111.

11. Najarian JS, SutherlandDE, MatasAJ, Steffes MW, Simmons RL, GoetzFC. Human islet transplantation: a preliminary report. Transplant Proc. 1977;9(1):233-236.

12. Largiadèr F, Kolb E, Binswanger U. A long-term functioning human pancreatic islet allotransplant. Transplantation. 1980;29(1):76-77.

13. Agarwal A, Brayman KL. Update on islet cell transplantation for type 1 diabetes. Semin Intervent Radiol. 2012;29(2):90-98.

14. Barton FB, Rickels MR, Alejandro R, et al. Improvement in outcomes of clinical islet transplantation: 1999-2010. Diabetes Care. 2012;35(7):1436-1445.

15. Barton F, Stablein D. CITR 8th Annual Report. Collaborative Islet Transplant Registry. Available from: http://www.citregistry.org. Accessed September 15, 2016

16. Cogger K, Nostro MC. Recent advances in cell replacement therapies for the treatment of type 1 diabetes. Endocrinology. 2014;156(1):8-15.

17. Shapiro AMJ. Islet transplantation in type 1 diabetes: ongoing challenges, refined procedures, and long-term outcome. Rev Diabet Stud. 2012;9(4):385-406.

18. Lablanche S, Borot S, Wojtusciszyn A, et al. Five-year metabolic, functional, and safety results of patients with type 1 diabetes transplanted with allogenic islets within the Swiss-French GRAGIL network. Diabetes Care. 2015;38(9):dc150094.

19. Hering BJ, Clarke WR, Bridges ND, et al. Phase 3 trial of transplantation of human islets in type 1 diabetes complicated by severe hypoglycemia. Diabetes Care. 2016;39(7):1230-1240.

20. Markmann JF, Deng S, Huang X, et al. Insulin independence following isolated islet transplantation and single islet infusions. Ann Surg. 2003;237(6):741-750.

21. Chhabra P, Sutherland DER, Brayman KL. Overcoming barriers in clinical islet transplantation: current limitations and future prospects. Curr Probl Surg. 2014;51(2):49-86.

22. Ramesh A, Chhabra P, Brayman KL. Pancreatic islet transplantation in type 1 diabetes mellitus: an update on recent developments. Curr Diabetes Rev. 2013;9(4):294-311.

23. Vantyghem M-C, Raverdy V, Balavoine A-S, et al. Continuous glucose monitoring after islet transplantation in type 1 diabetes: an excellent graft function ( $\beta$-score greater than 7 ) is required to abrogate hyperglycemia, whereas a minimal function is necessary to suppress severe hypoglycemia ( $\beta$-score greater than 3). J Clin Endocrinol Metab. 2012;97(11):E2078-E2083. doi:10.1210/jc.2012-2115.

24. Ryan EA, Paty BW, Senior PA, Lakey JRT, Bigam D, Shapiro AMJ. Beta-score: an assessment of beta-cell function after islet transplantation. Diabetes Care. 2005;28(2):343-347.

25. Rickels MR, Fuller C, Dalton-Bakes C, et al. Restoration of glucose counterregulation by islet transplantation in long-standing type 1 diabetes. Diabetes. 2015;64(5):1713-1718. doi:10.2337/db14-1620.

26. Rickels MR. Recovery of endocrine function after islet and pancreas transplantation. Curr Diab Rep. 2012;12(5):587-596.

27. Piemonti L, Everly MJ, Maffi P, et al. Alloantibody and autoantibody monitoring predicts islet transplantation outcome in human type 1 diabetes. Diabetes. 2013;62(5):1656-1664. 
28. Bosi E, Braghi S, Maffi P, et al. Autoantibody response to islet transplantation in type 1 diabetes. Diabetes. 2001;50(11):2464-2471.

29. Rickels MR, Kearns J, Markmann E, Palanjian M, Markmann JF, Naji A, Kamoun M. HLA sensitization in islet transplantation. Clin Transpl. 2006:413-420.

30. Kawahara T, Kin T, Kashkoush S, et al. Portal vein thrombosis is a potentially preventable complication in clinical islet transplantation. Am J Transplant. 2011;11(12):2700-2707.

31. Villiger P, Ryan EA, Owen R, et al. Prevention of bleeding after islet transplantation: lessons learned from a multivariate analysis of 132 cases at a single institution. Am J Transplant. 2005;5(12):2992-2998.

32. Ryan EA, Paty BW, Senior PA, et al. Five-year follow-up after clinical islet transplantation. Diabetes. 2005;54(7):2060-2069.

33. Jahansouz C, Jahansouz C, Kumer SC, Brayman KL. Evolution of $\beta$-cell replacement therapy in diabetes mellitus: islet cell transplantation. J Transplant. 2011;2011:1-21

34. Kumar R, Chung WY, Dennison AR, Garcea G. Current principles and practice in autologous intraportal islet transplantation: a metaanalysis of the technical considerations. Clin Transplant. 2016;30(4): 344-356.

35. Lee Y, Ravazzola M, Park B-H, Bashmakov YK, Orci L, Unger RH. Metabolic mechanisms of failure of intraportally transplanted pancreatic beta-cells in rats: role of lipotoxicity and prevention by leptin. Diabetes. 2007;56(9):2295-2301.

36. Venturini M, Maffi P, Querques G, et al. Hepatic steatosis after islet transplantation: Can ultrasound predict the clinical outcome? a longitudinal study in 108 patients. Pharmacol Res. 2015;98:52-59.
37. Leitão CB, Peixoto EML, Westphalen $\mathrm{AC}$, et al. Liver fat accumulation after islet transplantation and graft survival. Cell Transplant. 2014;23(10):1221-1227.

38. Bhargava R, Senior PA, Ackerman TE, Ryan EA, Paty BW, Lakey JR, Shapiro AM. Prevalence of hepatic steatosis after islet transplantation and its relation to graft function. Diabetes. 2004;53(5):1311-1317.

39. Targher G, Mantovani A, Pichiri I, et al. Nonalcoholic fatty liver disease is independently associated with an increased incidence of chronic kidney disease in patients with type 1 diabetes. Diabetes Care. 2014;37(6):1729-1736. doi:10.2337/dc13-2704.

40. Nathan DM. Long-term complications of diabetes mellitus. $N$ Engl $J$ Med. 1993;328(23):1676-1685.

41. Vantyghem M-C, Kerr-Conte J, Arnalsteen L, et al. Primary graft function, metabolic control, and graft survival after islet transplantation. Diabetes Care. 2009;32(8):1473-1478.

42. Bowers DT, Botchwey EA, Brayman KL. Advances in local drug release and scaffolding design to enhance cell therapy for diabetes. Tissue Eng Part B Rev. 2015;21(6):491-503.

43. Sabek OM, Farina M, Fraga DW, et al. Three-dimensional printed polymeric system to encapsulate human mesenchymal stem cells differentiated into islet-like insulin-producing aggregates for diabetes treatment. J Tissue Eng. 2016;7.

44. Song S, Roy S. Progress and challenges in macroencapsulation approaches for type 1 diabetes (T1D) treatment: Cells, biomaterials, and devices. Biotechnol Bioeng. 2016;113(7):1381-1402.

45. Chhabra P, Brayman KL. Stem cell therapy to cure type 1 diabetes: from hype to hope. Stem Cells Transl Med. 2013;2(5):328-336.
Diabetes, Metabolic Syndrome and Obesity: Targets and Therapy is an international, peer-reviewed open-access journal committed to the rapid publication of the latest laboratory and clinical findings in the fields of diabetes, metabolic syndrome and obesity research. Original research, review, case reports, hypothesis formation, expert opinion and commentaries are all considered for publication. The manuscript management system is completely online and includes a very quick and fair peer-review system, which is all easy to use. Visit http://www.dovepress.com/testimonials.php to read real quotes from published authors. 